\title{
Erratum zu: Vorzug Ganztagsschule? Zusammenhänge mit der Kompetenzentwicklung im Bereich Lesen und Mathematik
}

\author{
Tobias Linberg • Olaf Struck • Thomas Bäumer
}

Online publiziert: 4. März 2019

(C) The Editors of the Journal 2019

Erratum zu: Z Erziehungswiss (2018) 21:1205-1227

https://doi.org/10.1007/s11618-018-0830-2

Leider liegt in der Originalversion des Artikels ein Fehler vor: Der Drittautor Dr. Thomas Bäumer wurde versehentlich einer falschen Affiliation zugeordnet. Die korrekte Affiliationsangabe für Dr. Thomas Bäumer lautet:

Leibniz-Institut für Bildungsverläufe (LIfBi)

Wilhelmsplatz 3, 96047 Bamberg

Für diesen Fehler bitten wir um Entschuldigung.

Die Online-Version des Originalartikels ist unter https://doi.org/10.1007/s11618-018-0830-2 zu finden.

Dr. T. Linberg $(\bowtie)$

Staatsinstitut für Schulqualität und Bildungsforschung, Schellingstraße 155, 80797 München, Deutschland

E-Mail: tobias.linberg@isb.bayern.de

Prof. Dr. O. Struck

Professur für Arbeitswissenschaft, Otto-Friedrich-Universität Bamberg,

Feldkirchenstraße 21, 96052 Bamberg, Deutschland

E-Mail: olaf.struck@uni-bamberg.de

Dr. T. Bäumer

Leibniz-Institut für Bildungsverläufe (LIfBi), Wilhelmsplatz 3, 96047 Bamberg, Deutschland

E-Mail: thomas.baeumer@lifbi.de 